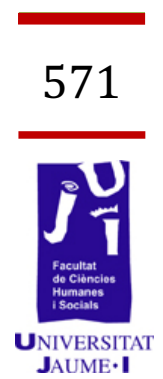

\title{
Las fuentes de información y el lenguaje en las agencias de comunicación científica. El caso de SINC
}

Susana Barberá Forcadell sbarbera@uji.es 
La comunicación de los avances en la ciencia se ha generalizado en algunos medios de comunicación del tal forma que las informaciones científicas ya no tienen solo como destino final las revistas especializadas. Pero esta generalización de la difusión de la ciencia es una tarea que compete tanto a los investigadores como a los periodistas. A los primeros para que sepan hacer llegar de una manera más clara sus investigaciones confiando en los periodistas y a los segundos profesionalizándose en esta área del periodismo. Esta investigación analiza de qué manera se lleva a cabo la difusión de la comunicación científica por parte de los científicos y también de los periodistas. Para ello se analiza una agencia de información de reciente creación como es la plataforma del Servicio de Información y Noticias Científicas (SINC) teniendo en cuenta que su nacimiento data del año 2008. En la investigación se analizan entre otros dos aspectos fundamentales para conocer cómo se elabora y traslada esta información a la sociedad. De un lado cómo son las fuentes de información más utilizadas por los periodistas que se dedican a esta especialidad y de otro qué tipo de lenguaje es el empleado, puesto que en general el científico utiliza muchos tecnicismos. Dicho análisis confirma que las fuentes de información a las que se dirigen los periodistas para escribir sobre ciencia en SINC son casi todas personales y además claramente especializadas, ya sean científicos o personal docente e investigador de una universidad y que el lenguaje es bastante claro ya que la mayoría de los términos técnicos empleados se explican mediante definiciones simples que facilitan la comprensión del texto por parte del lector, a pesar de ello todavía se refleja un elevado número de piezas informativas con lenguaje confuso que impide su total comprensión.

Palabras clave: comunicación, periodismo científico, divulgación de la ciencia, tecnicismos, fuentes de información, agencia SINC

\section{Introducción}

La comunicación de la ciencia se ha realizado desde los inicios de ésta a través de los propios científicos mediante sus divulgaciones. Pero esta tarea se ha profesionalizado con el paso de los años con la aparición del periodismo científico cuya finalidad es transmitir a la sociedad los avances de la ciencia de una forma clara para su mejor comprensión.

Este trabajo tiene como finalidad analizar las informaciones sobre ciencia que se publican en una agencia de noticias especializadas como es el Servicio de Información y Noticias Científicas (SINC), la primera agencia pública española de estas características. Para ello se ha elegido como muestra los cuatro anuarios publicados por la agencia para estudiar qué géneros son los más utilizados, cuál es el origen de las informaciones, a qué fuentes se recurre, cuáles son los temas de los que más se publica y qué tipo de lenguaje se emplea. 
La necesidad de conocer los avances que se producen de forma continuada por los científicos, en cualquier campo en el que se dé, es básica, aunque no es un hecho que se realiza de forma natural en los medios de comunicación de masas. El tema de este trabajo de investigación surgió tras trabajar durante varios años en el Instituto Interuniversitario de Desarrollo Local (IIDL) de la Universitat Jaume I dedicado a la investigación y la docencia. El trabajo realizado era como periodista y por lo tanto la persona que se tenía que encargar de realizar la difusión de las actividades que se llevaban a cabo dentro de dicho grupo.

En el cometido de este trabajo se redactaron informaciones sobre numerosas actividades organizadas desde el citado órgano universitario con el objetivo de darlas a conocer. Esto se pudo realizar con la ayuda del Servicio de Comunicación de la universidad, puesto que cada una de las notas de prensa elaboradas se enviaba a todos los medios de las listas de distribución del centro académico. Además otra de las funciones era organizar y difundir talleres, seminarios y conferencias que se realizaron durante la estancia.

La idea inicial del trabajo era analizar la difusión que se realizaba por parte de los grupos de investigación, pero visto que el tema de la difusión de la ciencia por estos grupos es muy amplio, dado que en él se pueden incluir desde las actividades que llevan a cabo como pueden ser los congresos, talleres, seminarios hasta los artículos en prensa y otro tipo de difusión, lo que correspondería más al área de Comunicación Corporativa, el objetivo de este trabajo se ha centrado en un aspecto específico más especializado en el área de Periodismo, como es el análisis de las piezas informativas publicadas.

Por ello, este trabajo se centra en una idea definida como es, tomando como referencia el "Anuario SINC. La ciencia es noticia", un anuario que se elabora a partir de las noticias publicadas en la web www.agenciasinc.es que nació el 7 de febrero de 2008, analizar el tratamiento periodístico de todas las informaciones que se han recogido en el mismo. El Servicio de Información y Noticias Científicas (SINC) es la primera agencia pública de ámbito estatal especializada en información sobre ciencia, tecnología e innovación en español, dependiente de la Fundación Española para la Ciencia y la Tecnología (FECYT) del Ministerio de Economía y Competitividad del Gobierno de España.

La particularidad con la que cuenta este Servicio es que solamente realiza sus publicaciones diarias en Internet, aunque posteriormente se encargue de compilar las mejores informaciones en un anuario que se publica un mes después de acabado el año, tras el trabajo de selección de los artículos. Una curiosidad de este trabajo anual que desarrolla se refiere a que la agencia no comenzó a elaborar dicho anuario hasta el año 2010 por lo que los años 2008 y 2009 no cuentan con una publicación impresa en la que se muestran las informaciones más relevantes publicadas en la web.

A pesar de su joven andadura, la agencia es un referente importante como fuente de información científica en español y su alcance sigue 
creciendo, tal y como demuestra el incremento del $32 \%$ en el número de visitas que recibió la web durante el año 2012 según Google Analytics. Estos datos vienen reflejados en la publicación de dicho anuario, destacando además que las visitas a SINC no solo proceden de España, sino que también tienen su origen en otros países como es el caso de Estados Unidos, México, Colombia, Argentina, Perú, Chile, Venezuela o Ecuador por citar algunos.

Tal como explican desde la agencia, SINC nació con la misión de ser un puente entre la ciencia y la sociedad y seis años después de su nacimiento, desde SINC afirman que está logrando visibilizar la ciencia que se hace en España, dotando a los periodistas de recursos para que la voz de la ciencia se refleje en los medios de comunicación y llegue de esta forma a toda la sociedad, por ello aseguran que el trabajo que realizan como agencia web ha contribuido y seguirá haciéndolo a incrementar la cultura científica de la sociedad en la que vivimos.

Todo esto se traduce, siempre según la agencia, en un incremento notable del número de visitantes, visitas y páginas vistas en la web. Con cerca de tres millones de visitantes, más de tres millones y medio de visitas y casi seis millones de páginas vistas, desde la agencia confirman que sin duda la ciencia es noticia.

\section{Objetivos}

Los objetivos generales de este trabajo pretenden descubrir cómo se realiza la difusión de la comunicación científica. Para ello se analizará una fuente de información bastante novedosa como es la plataforma del Servicio de Información y Noticias Científicas (SINC), teniendo en cuenta que su nacimiento data del año 2008. Esta agencia de noticias científicas, como también es conocida, se fundó el 7 de febrero de 2008 por la Fundación Española para la Ciencia y la Tecnología (Fecyt), dependiente del Ministerio de Ciencia y Tecnología. Es importante destacar que este análisis se fundamenta en 166 artículos publicados en los cuatro anuarios en papel que ha publicado el SINC desde el año 2010, fecha en la que publicó el primer anuario del Servicio de Información y Noticias Científicas, hasta el año 2013, cuyo volumen se imprimió en el mes de enero de 2014.

Los objetivos en los que se centra la investigación son:

1. Conocer la utilización de las fuentes de información en los artículos publicados y analizados. En este punto se ha estudiado cuántas hay, de qué naturaleza son y los tipos específicos con los que se trabajan.

2. Cuantificar la utilización de tecnicismos en la redacción de los artículos sobre ciencia en estos anuarios, frente a lo que se considera un lenguaje claro, es otra de las claves que tiene como objetivo este trabajo. 
El objeto de estudio de este trabajo es el tratamiento informativo del periodismo científico en el Servicio de Información y Noticias Científicas (SINC) -también denominada agencia SINC- que es la primera agencia pública de ámbito estatal especializada en información sobre ciencia, tecnología e innovación en español.

La definición de la muestra a emplear para acometer este trabajo han sido los anuarios publicados por la agencia SINC. Su elección se basa en disponer de toda la información publicada por esta agencia en formato papel para analizar de una forma más profunda y directa las piezas que se han escrito sobre ciencia los periodistas de esta agencia, que tiene carácter público y estatal, por lo que llega a todos los medios de comunicación, tanto españoles como internacionales. El hecho de elegir esta muestra y no otra se ha debido a la selección, por parte de SINC, de unos artículos determinados que son los que aparecen publicados en papel impreso en los anuarios. Esto determina la selección de los redactores de la citada agencia de unas piezas informativas, frente al total que se publica durante todo un año en la web. Esto supone una valoración de estas piezas que aparecen en los anuarios impresos, como muestra exponente de todo el trabajo que realiza el equipo de periodistas del Servicio de Información y Noticias Científicas.

En concreto, la muestra analizada se basa en los anuarios, en papel impreso, de los años 2010, 2011, 2012 y 2013 del Servicio de Información y Noticias Científicas (SINC). Según informa el gabinete de Comunicación de dicha agencia la recopilación de las mejores noticias, reportajes y entrevistas más destacados que han ido publicando durante todo un año lo imprimen a finales del mes de enero del año siguiente para poder difundirlo en papel a todas las personas que lo soliciten.

Cada uno de los anuarios contiene un número diferente de informaciones, así como de géneros periodísticos que se ha podido contabilizar de antemano para luego analizar de una forma detallada a través de una plantilla de análisis de contenido.

La unidad de recogida de datos es cada una de las piezas informativas que aparecen publicadas en los cuatro anuarios de la agencia. Este término engloba todas las informaciones que se encuentran en los cuatro volúmenes citados, a excepción en el último anuario -el correspondiente al año 2013- a las noticias debido a que se han publicado en formato de noticia breve y no se pueden analizar bajo el mismo parámetro que el resto de las informaciones, al tener menos categorías a analizar, por lo que los resultados de la muestra general no serían los correctos. 
Para abordar la exposición de resultados fruto del análisis de contenido realizado a las piezas informativas publicados en los anuarios de la agencia SINC de los años 2010, 2011, 2012 y 2013 se han tomado como grandes áreas de resultados las cuestiones más destacadas para poder realizar una valoración respecto a los objetivos planteados.

\subsection{Tratamiento de las fuentes en la pieza informativa}

El análisis del tratamiento de las fuentes de información en la pieza informativa se lleva a cabo a partir del número de las mismas, de la naturaleza que tengan y en tercer lugar del tipo específico de fuente de que se trate para saber quién ofrece esta información al periodista.

\subsubsection{Número de fuentes de información}

Las fuentes de información son clave para elaborar la pieza informativa. Las informaciones analizadas muestran un equilibrio (ver Gráfico 1) en la utilización de una fuente (50 piezas) y de dos fuentes (57), aunque predomina ligeramente la segunda opción. Destaca la empleabilidad de más de tres fuentes (en 40 de los artículos publicados). La opción de no citar ninguna fuente se encuentra en cuatro informaciones pero es debido a la aparición del género efemérides en el último de los anuarios publicados.

En cuanto a los resultados de los análisis de las fuentes de información por años (ver Gráfico 2) llama la atención el mayor uso de dos fuentes en las piezas redactadas en los anuarios de 2010 y 2011 . Este predominio cambia de forma radical en los dos años siguientes, puesto que tanto en 2012 como en 2013 son predominantes (con 16 cada una) las piezas que se han elaborado a partir de una y de más de tres fuentes de información. Llama la atención que los artículos escritos a partir de dos y tres fuentes respectivamente comparten el mismo número de publicaciones (7 en el año 2012) mientras descienden de una forma muy acusada en 2013 con tan solo dos artículos de dos fuentes y uno de tres en 2013, apareciendo un elemento distinto del resto de los anuarios, que es la publicación de cuatro piezas guardando el anonimato del informante. Esto es debido a las efemérides. 
Gráfico 1. Fuentes de información

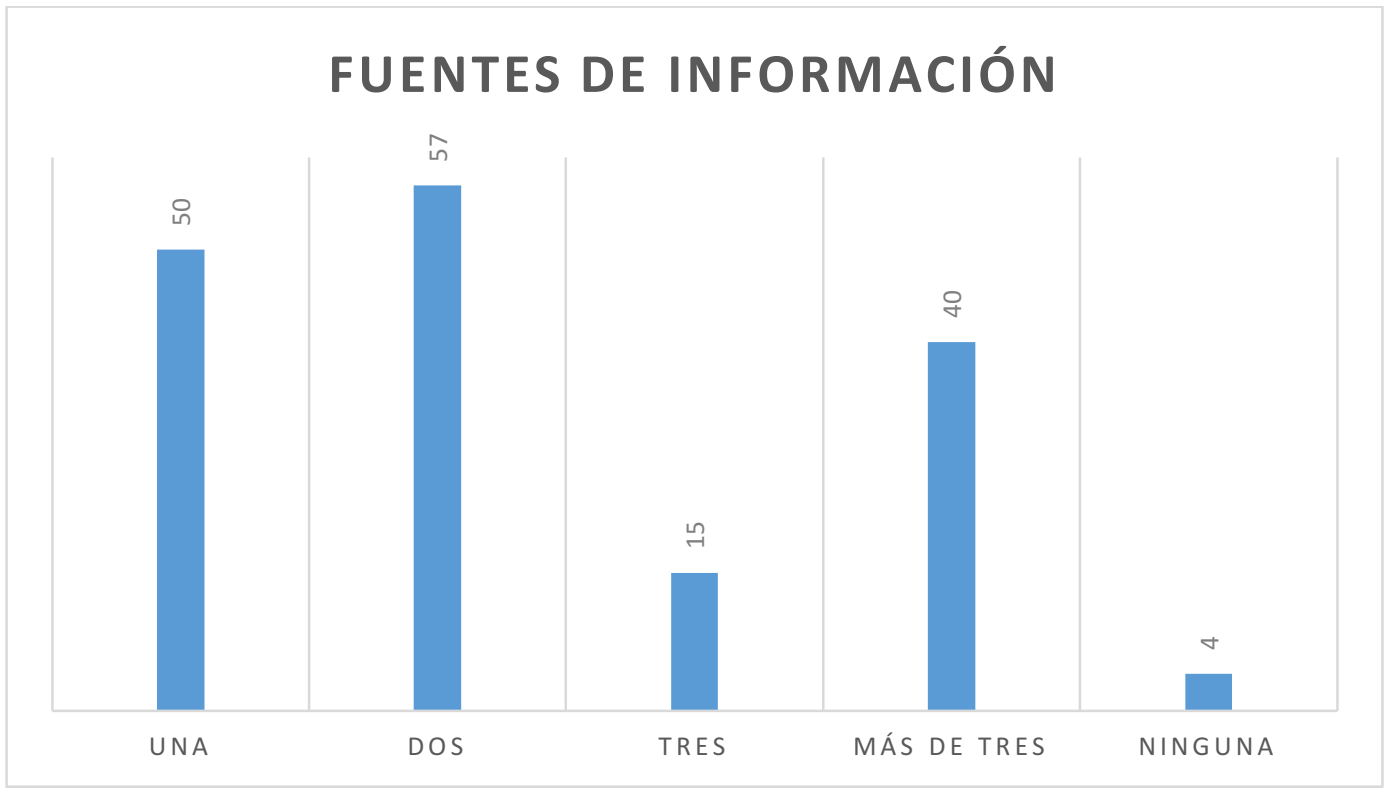

Fuente: Elaboración propia

Gráfico 2. Fuentes de información. Por años.

Fuentes de información. Por años

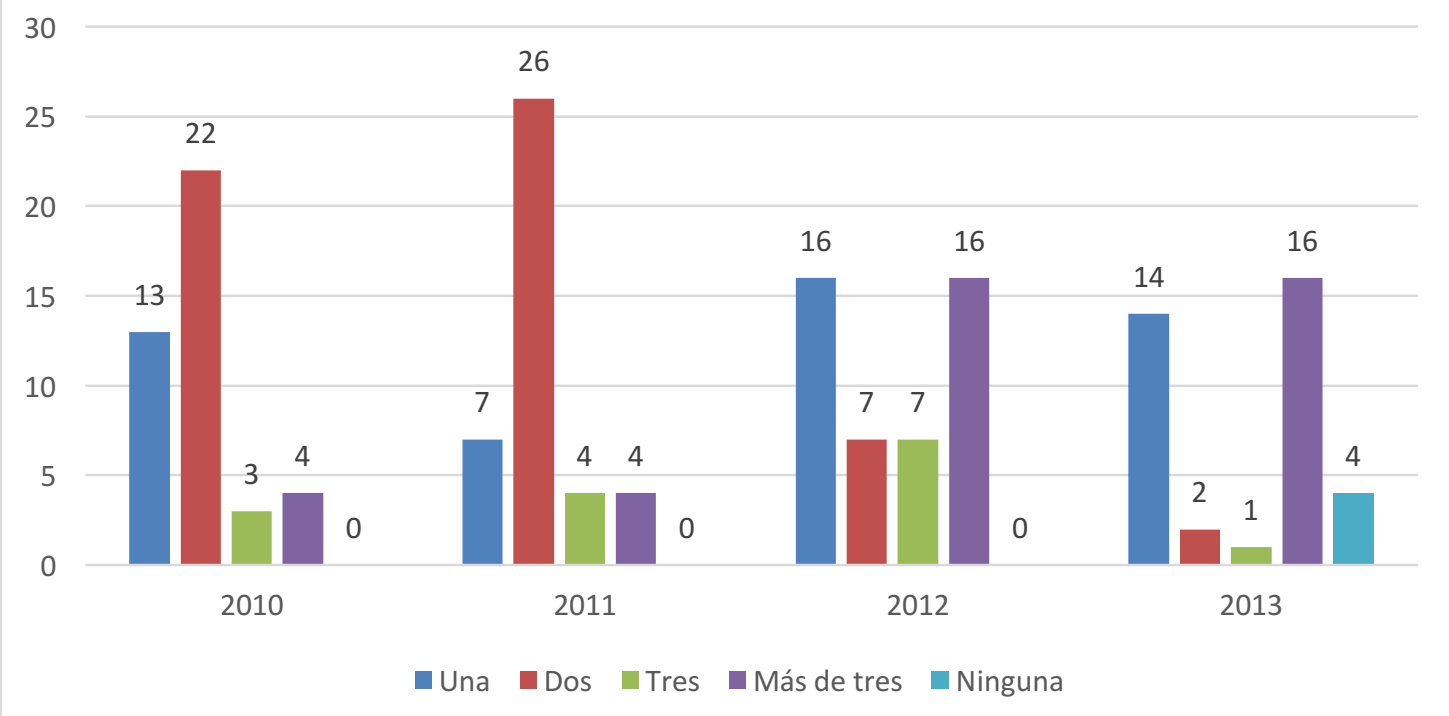

Fuente: Elaboración propia 


\subsubsection{Naturaleza de las fuentes}

El resultado que arroja el análisis de la naturaleza de las fuentes de información empleadas es de un predominio del uso de las fuentes personales frente a las documentales (ver Gráfico 3). Hay 158 personas a las que se ha preguntado para elaborar las informaciones (el 65\%) frente a 86 fuentes de carácter documental (35\%).

En el análisis de la naturaleza de las fuentes por años (ver Tabla 1) se aprecia una progresiva disminución de las fuentes informativas, especialmente las documentales, desde los inicios en 2010 hasta el último anuario. Así, mientras el primer anuario contenía 28 fuentes documentales se ha llegado a la mitad en el año 2013. En cuanto a las fuentes personales, la bajada también se ha notado, a pesar de que no de una forma tan drástica como ha sido con las documentales. En este sentido, en el primer anuario comenzaron empleando 41 fuentes personales para redactar sus piezas informativas y en el último han bajado a 32 fuentes.

Gráfico 3. Naturaleza de las fuentes

\section{Naturaleza de las fuentes}

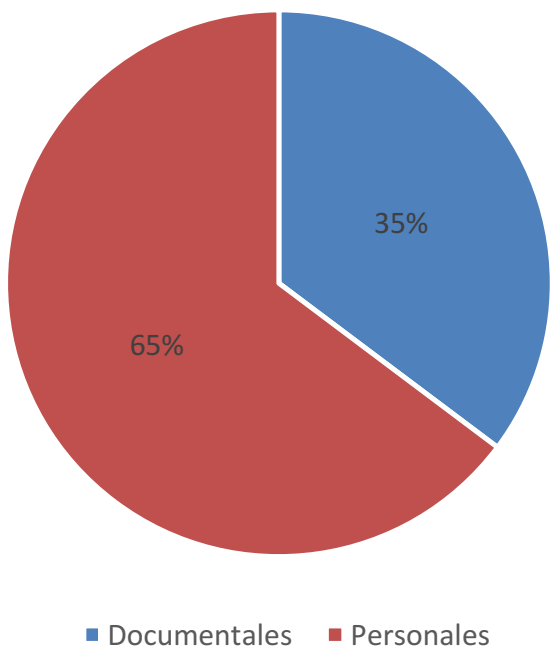

Fuente: Elaboración propia

Tabla 1. Naturaleza de las fuentes. Por años

\begin{tabular}{|l|c|c|c|c|c|}
\hline & $\mathbf{2 0 1 0}$ & $\mathbf{2 0 1 1}$ & $\mathbf{2 0 1 2}$ & $\mathbf{2 0 1 3}$ & TOTAL \\
\hline Documentales & 28 & 27 & 17 & 14 & 86 \\
\hline Personales & 41 & 40 & 45 & 32 & 158 \\
\hline
\end{tabular}

Fuente: Elaboración propia 


\subsubsection{Tipos específicos de fuentes}

El resultado en los tipos específicos (ver Gráfico 4) es de una gran diferencia entre el uso de las fuentes científicas (95) y autoridades o cargos universitarios (79) respecto al resto de fuentes empleadas.

Por lo que respecta a la comparativa por años (ver Gráfico 5) se observa un equilibrio de la utilización de las fuentes científicas (15 y 21) como de las de autoridad o cargo universitario ( 25 en ambos), en las informaciones aparecidas en los anuarios 2010 y 2011 . Y el siguiente año, en 2013, las piezas incluidas contienen más fuentes científicas (36) frente a la caída que suponen las de cargo universitario (13). Esta tendencia sigue, pero en clara bajada en el último año analizado, puesto que mientras hay 23 científicas, las de autoridad o rango universitario sube ligeramente hasta situarse en 16.

Gráfico 4. Tipos específicos de fuentes

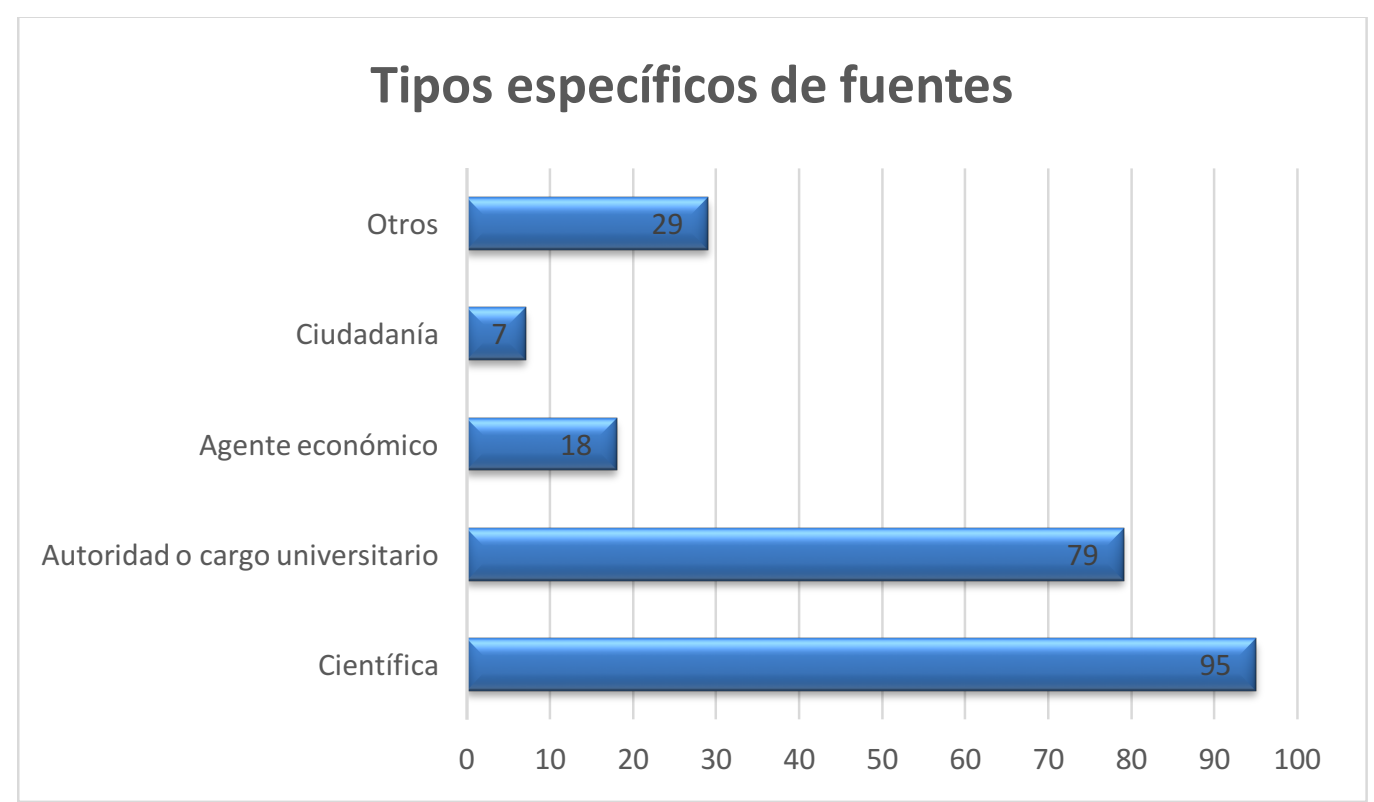

Fuente: Elaboración propia 
Gráfico 5. Tipos específicos de fuentes. Por años

Tipos específicos de fuentes. Por años

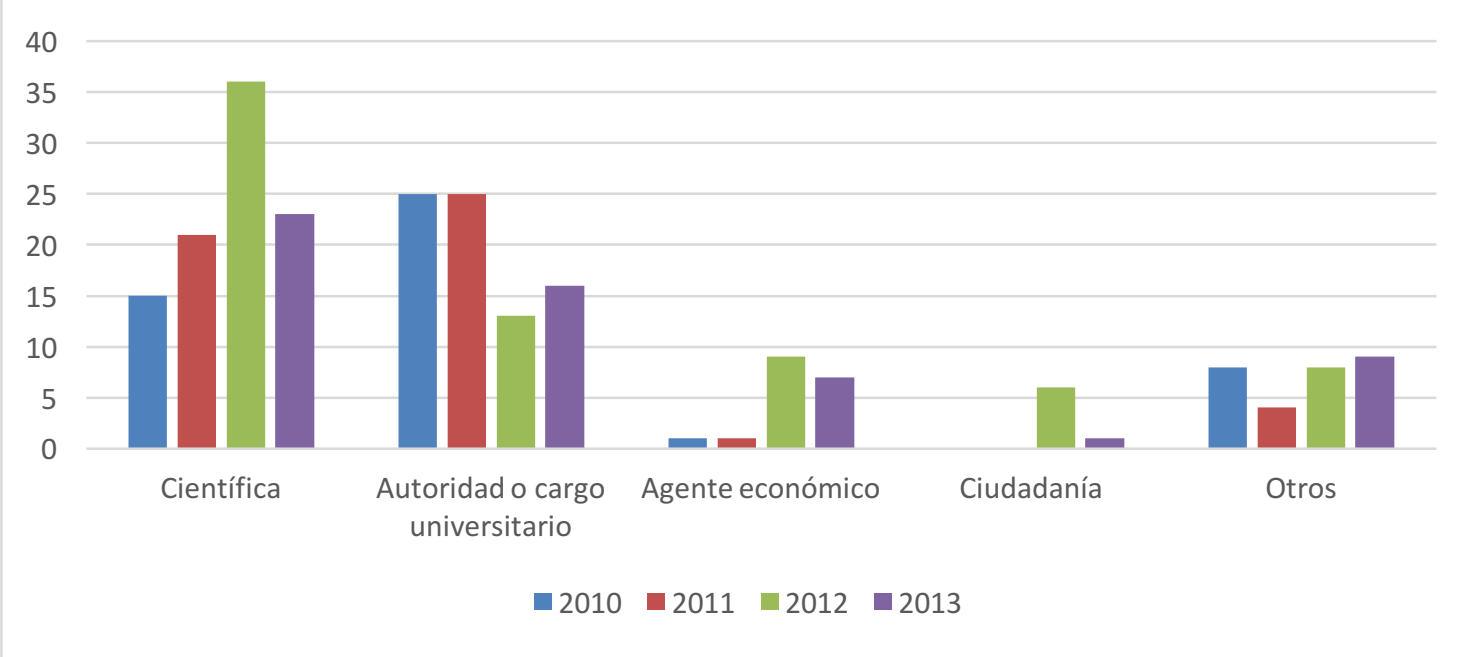

Fuente: Elaboración propia

\subsection{Lenguaje empleado en las piezas}

Para abordar el lenguaje empleado en las piezas informativas se analizan varios parámetros relativos a la utilización de los tecnicismos o no en los artículos publicados en los cuatro anuarios estudiados. En primer lugar se analiza el número de palabras técnicas empleadas, agrupadas en cantidades concretas, y después se ha realizado un análisis para conocer si se explican los tecnicismos empleados en los artículos, y en el caso de que se expliquen, en otro apartado se analiza cómo se hace dicha explicación. Todo esto y bajo unas premisas establecidas lleva a realizar la última parte de este apartado que es definir el tipo de lenguaje del documento como claro, técnico o por el contrario confuso.

\subsubsection{Número de tecnicismos en las piezas informativas}

La cantidad de palabras especializadas sobre el tema del que se está hablando en la información es fundamental a la hora de evaluar posteriormente la dificultad que entrañará el texto para el lector a la hora de su comprensión. El lenguaje empleado en las piezas informativas analizadas tiene un elevado número de tecnicismos, entendido como un conjunto de voces técnicas empleadas en el lenguaje de un arte, de una ciencia, de un oficio, etc.

Tras realizar el análisis de los artículos seleccionados (ver Gráfico 6) se ha constatado que la mayoría de estos, 60 informaciones, tienen entre 1 y 2 tecnicismos. Siguiendo este orden de importancia en número, en segundo lugar se sitúan aquellos artículos que no contienen ningún tecnicismo, un total de 48 , lo que significa que el lenguaje empleado en el texto tiene una fácil comprensión para el lector y el tercer lugar lo ocupan las piezas que contienen entre 3 y 5 palabras especializadas en el lenguaje del que se habla con 44 piezas que asumen estas características. El último puesto en esta lista, tras realizar el análisis, lo ocupan aquellas 
informaciones que contienen 6 o más tecnicismos, con 14 piezas, una cantidad muy inferior a la primera pero que muestra la presencia de este tipo de lenguaje tan específico en un tipo de periodismo muy especializado como es el periodismo científico.

En la valoración por años (ver Gráfico 7) se detecta un incremento moderado de las gráficas en las informaciones con 1 o 2 tecnicismos durante los tres primeros anuarios pasando de 14 el primer año a 16 y 18 en el tercero pero para descender en el último volumen con tan solo 12 piezas informativas con palabras muy especializadas. Otra categoría que experimenta un cambio a lo largo del estudio en los cuatro anuarios es la de los artículos con entre 3 y 5 tecnicismos, ya que comienza con 13 piezas que tienen esta cantidad para bajar hasta 5 en el último anuario de la agencia. También sufre una subida considerable la categoría de 6 o más tecnicismos en la información al pasar de 2 piezas en el primer anuario, 1 en el segundo, 5 en el tercero a 6 en el último, cantidad que aunque no supone un incremento muy destacable sí que se constata un constante crecimiento durante los cuatro años analizados.

Gráfico 6. Número de tecnicismos

\section{NÚMERO DE TECNICISMOS}

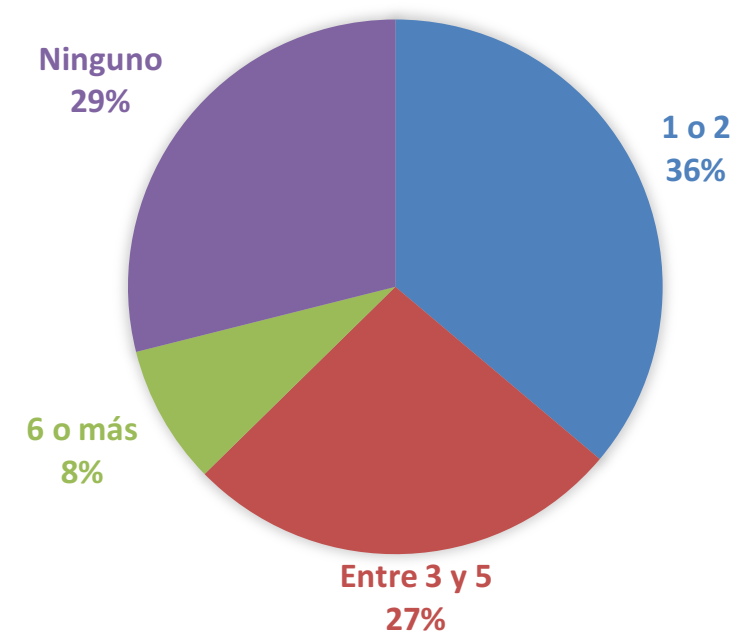

Fuente: Elaboración propia 
Número de tecnicismos. Por años

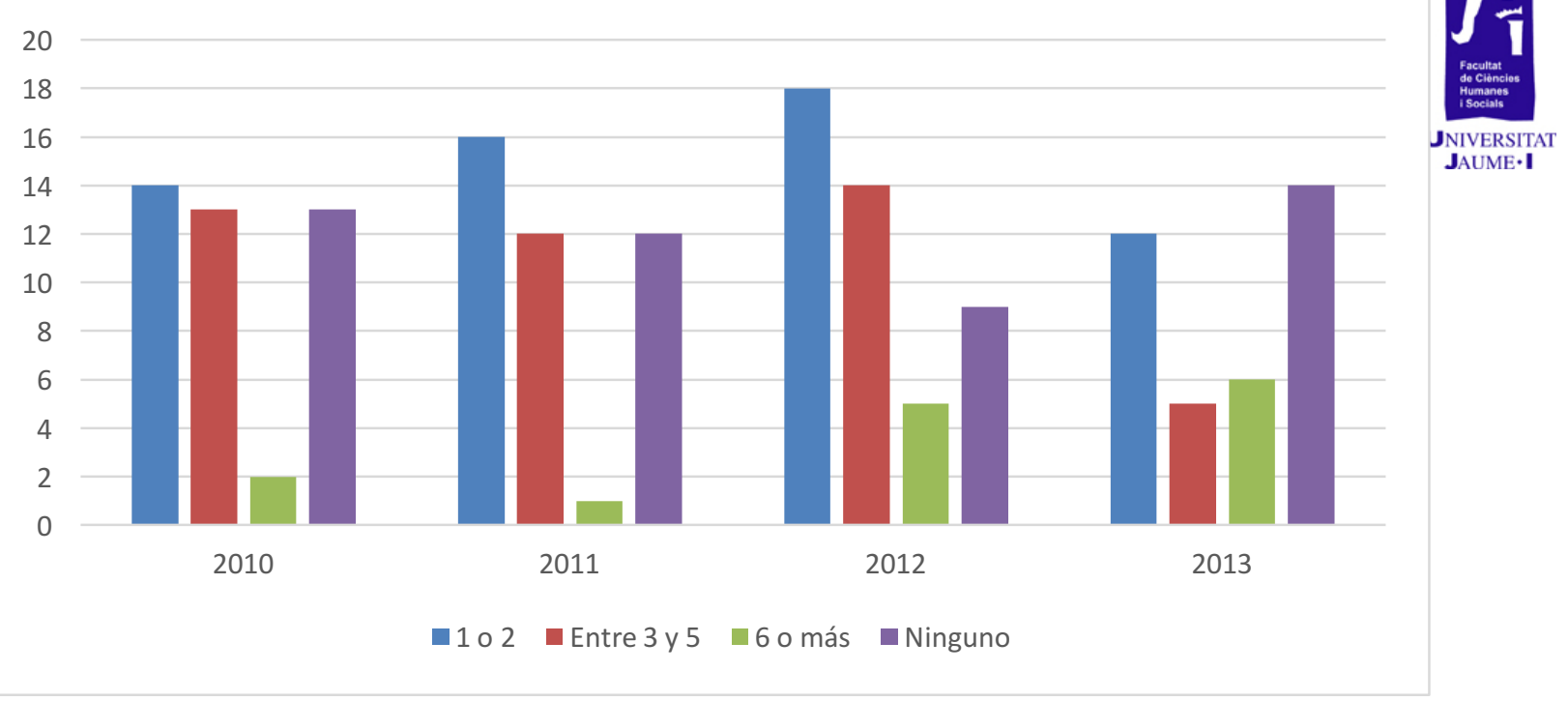

Fuente: Elaboración propia

\subsubsection{Explicación de los tecnicismos en la pieza informativa}

El análisis de la explicación de los tecnicismos en cada una de las informaciones publicadas permite observar que grado de comprensión pretende dar el redactor que elabora la información al lector. Los resultados de este apartado (ver Gráfico 8) muestran que la mayoría de los tecnicismos empleados sí que se explican, aunque sea en algunos casos. En este sentido, destaca que del total de los tecnicismos de los artículos hay un $57 \%$ de los artículos en los que sí que se explican todos los tecnicismos por parte de la persona que ha redactado la pieza informativa, frente a un $30 \%$ en los que se explican solo algunas las palabras especializadas aparecidas en la información. Por el contrario todavía queda un $13 \%$ de artículos con tecnicismos en los que no se realiza ningún tipo de definición sobre el término específico utilizado, lo que dificulta la comprensión del texto.

En el nivel de análisis por años destaca un incremento, durante los tres primeros anuarios de la explicación de este tipo de términos pasando de 13 a 23 en el año 2012, cifra que cae a 14 en el último volumen. También es significativo el descenso que experimenta en la gráfica el hecho de haya cada vez menos piezas informativas en las que no se expliquen de ninguna manera todos los tecnicismos que aparecen en las mismas, pasando de 6 el primer año a 2 el último (ver Gráfico 9). En cambio, aquellas piezas en las que se explican de alguna manera algunos de los tecnicismos aparecidos se mantienen relativamente puesto que pasan de 10 el primer año analizado a 7 en el cuarto anuario. 


\section{EXPLICACIÓN DE TECNICISMOS}

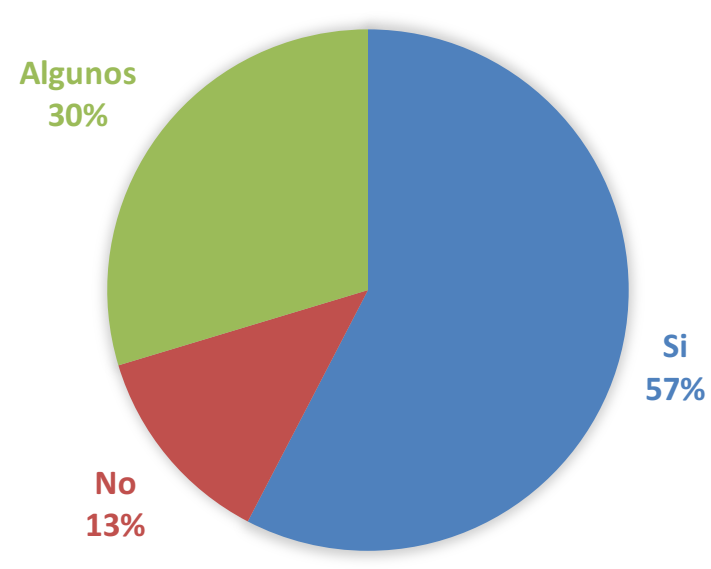

Fuente: Elaboración propia

Gráfico 9. Explicación de tecnicismos. Por años

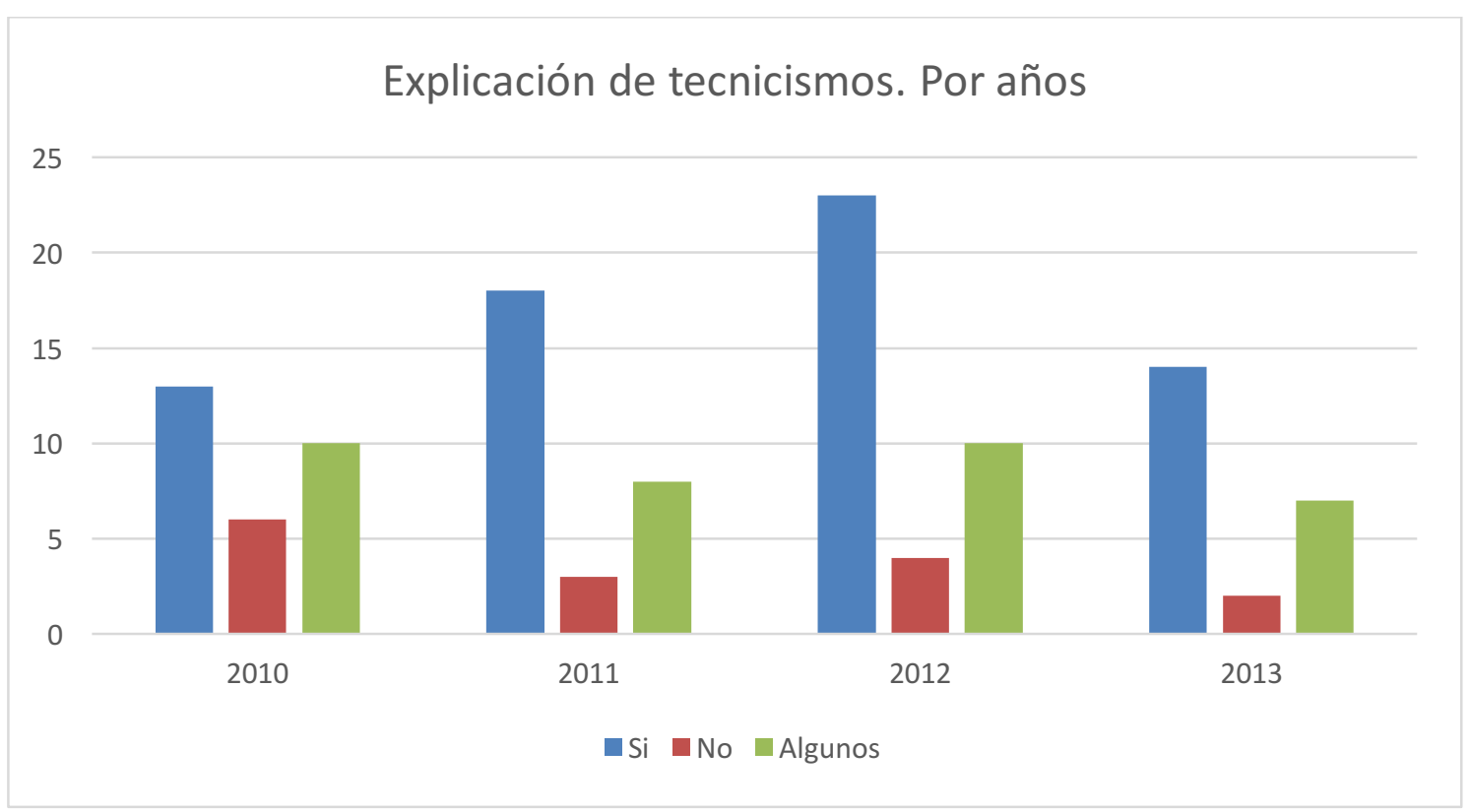

Fuente: Elaboración propia

\subsubsection{Cómo explica los tecnicismos}

La explicación de los tecnicismos define, como complemento al punto anterior, el grado de intento de comprensión de los términos empleados por parte del redactor. De esta forma se puede realizar de varias formas empleando otros tecnicismos, lo que dificulta de nuevo la comprensión de la información, con definiciones simples para facilitar la lectura del mismo 
o mediante otras explicaciones que no siendo definiciones pueden ayudar a su entendimiento.

En este apartado hay una clara voluntad (ver Gráfico 10) por parte de los redactores que han elaborado las informaciones de que los textos sean de fácil comprensión puesto que el 91\% de los tecnicismos (94 términos) se explican con definiciones simples, frente al $7 \%$ ( 7 palabras especializadas) cuya definición se vuelve a realizar con otros tecnicismos y tan solo el $2 \%$ ( 2 palabras) se basa en otro tipo de apoyos para explicarlas.

El análisis por años (ver Gráfico 11) muestra la mayoría de las informaciones en los anuarios analizados en los que hay una explicación de los tecnicismos con definiciones simples, incrementándose de una manera exponencial en el tercer anuario con 32 artículos en los que se explican de este modo. Por el contrario la definición con otro tipo de tecnicismos se mantiene por igual en cada año analizado manteniéndose en 2 en el primero, 2 en el segundo, para bajar a 1 en el tercero y 2 en el último volumen.

Gráfico 10. Cómo explica los tecnicismos

\section{CÓMO EXPLICA LOS TECNICISMOS}

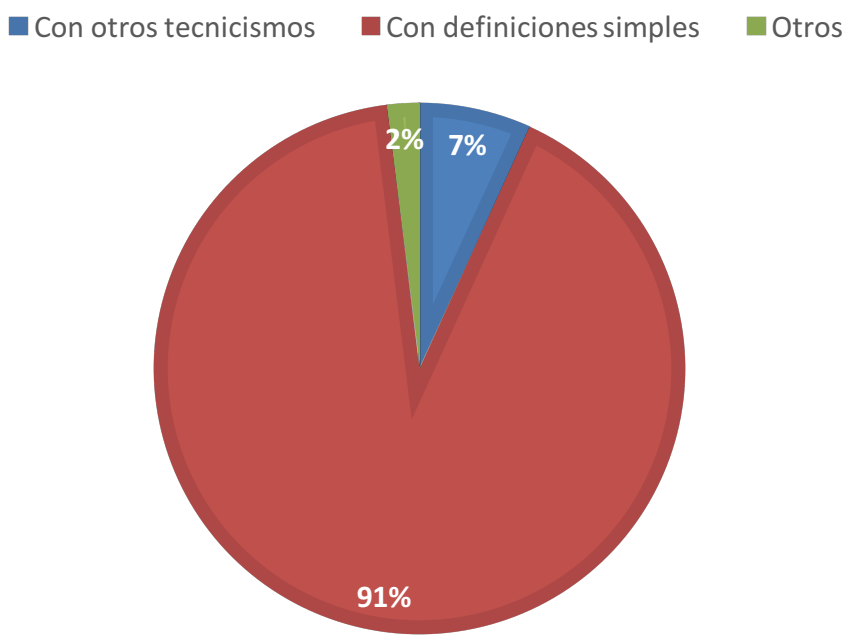

Fuente: Elaboración propia 


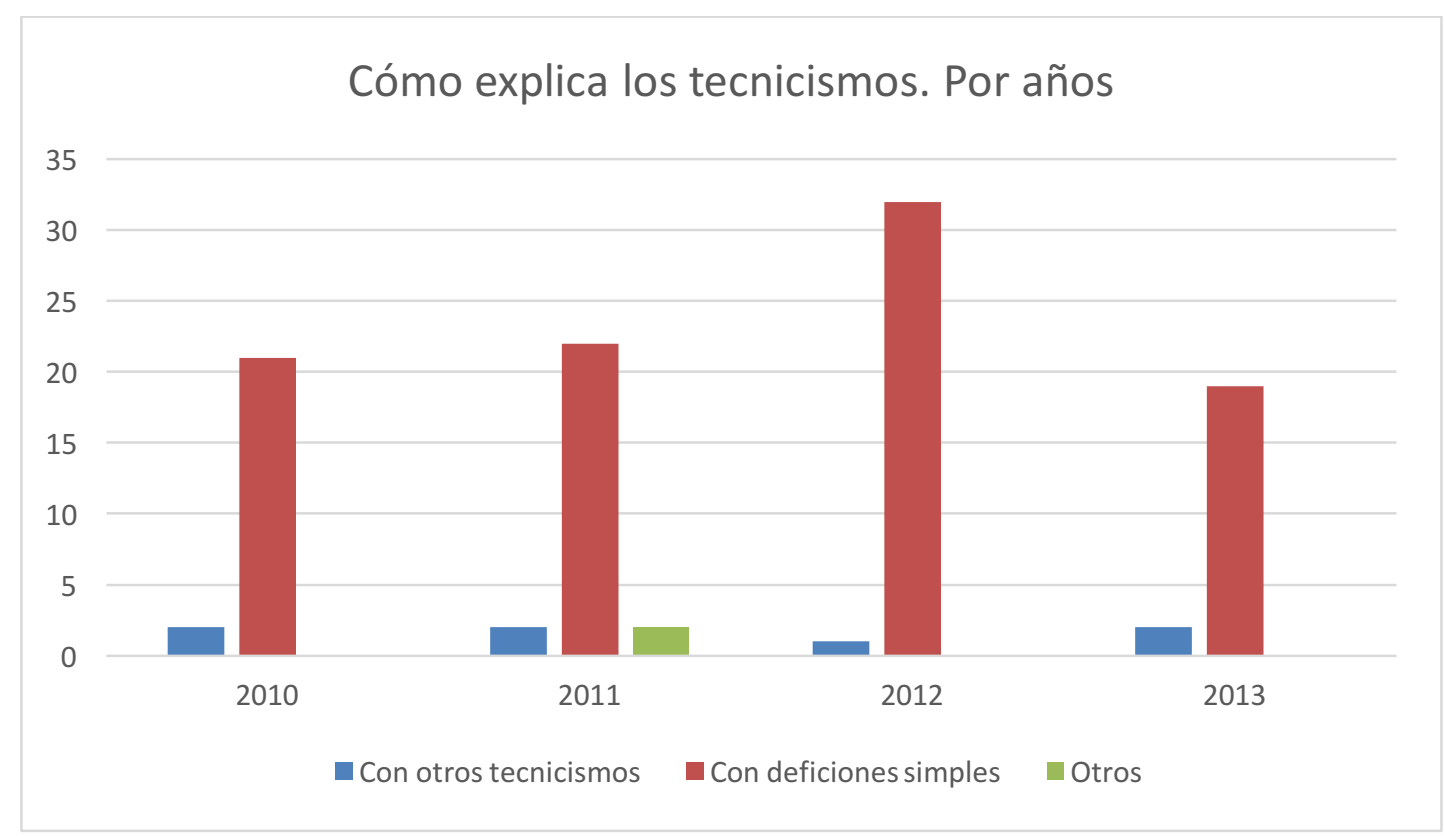

Fuente: Elaboración propia

\subsubsection{Lenguaje del documento según el número de tecnicismos empleados}

El último apartado analizado muestra abiertamente qué tipo de lenguaje es el empleado por parte de los redactores teniendo en cuenta las dos categorías expuestas anteriormente en esta exposición de resultados, ya que si se han explicado los tecnicismos con definiciones simples será catalogado como claro si tiene de 0 a 2 y los explica, será técnico si hay más de 3 pero los explica y confuso si hay tecnicismos y no los explica.

Bajo estas premisas, el resultado obtenido del análisis realizado muestra (ver Gráfico 12) que el 67\% de las informaciones (112 artículos) se pueden considerar claras puesto que carecen de estos términos específicos o tienen 1 o 2 y los explican con definiciones simples. Pero destaca el hecho todavía el $29 \%$ (48 piezas informativas) son consideradas confusas al tener tecnicismos y no explicarlos o hacerlo con otros tecnicismos. Todo ello frente a tan solo un $4 \%$ ( 6 informaciones) que se catalogan bajo el nombre de técnicas por tener más de 3 tecnicismos pero explicarlos.

El análisis por años muestra una clara tendencia hacia el aumento por intentar clarificar las informaciones sobre ciencia, puesto que el número de informaciones clasificadas como claro va en aumento durante los tres primeros anuarios con 23 en el primero, 29 en el segundo y 32 en el tercero a pesar de que en el cuarto experimenta una ligera bajada a 28. En este mismo gráfico se puede ver una tendencia a la disminución de piezas consideradas como confusas al pasar de 15 piezas en el año 2010 a 9 en el último anuario analizado, el de 2013 (ver Gráfico 13). 


\section{Lenguaje según los tecnicismos empleados}

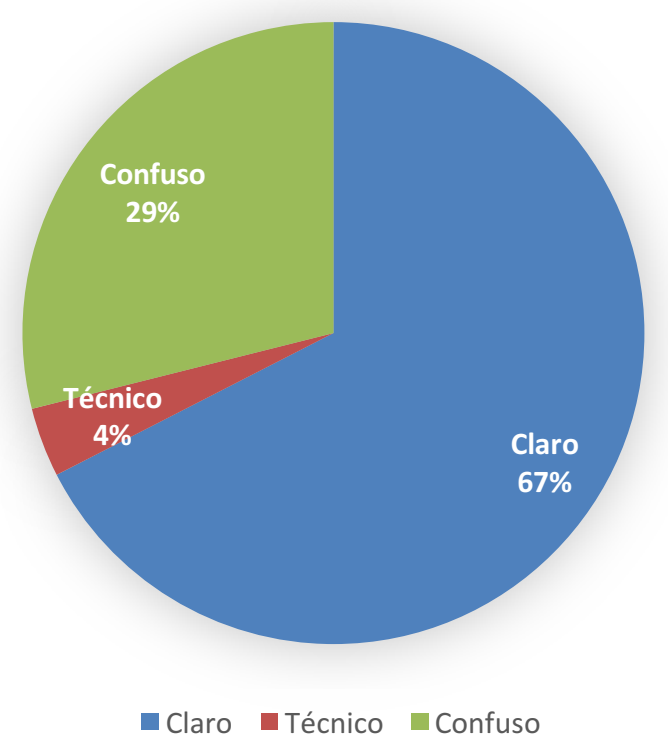

Fuente: Elaboración propia

Gráfico 13. Lenguaje según los tecnicismos empleados. Por años

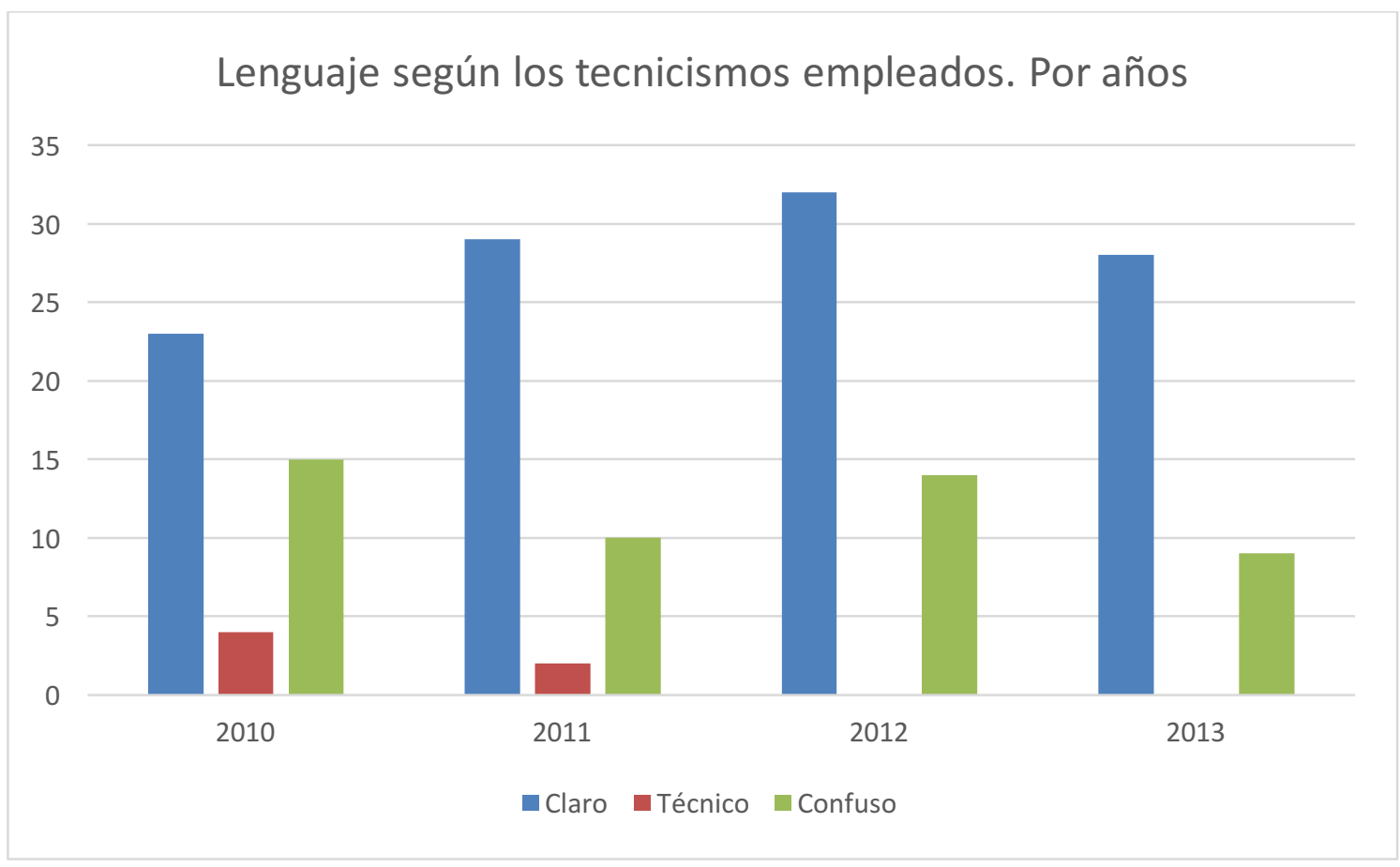

Fuente: Elaboración propia 


\section{Discusión y Conclusiones}

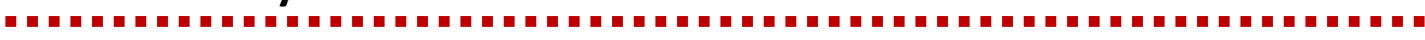

El análisis empírico de la difusión de la ciencia a través del Servicio de Información y Noticias Científicas (SINC), realizado en los anteriores apartados, permite obtener una serie de conclusiones con unas características muy definidas. A continuación se expone una clasificación de las conclusiones más relevantes obtenidas tras el citado análisis.

a. El género periodístico que domina en las informaciones científicas publicadas es la noticia, a pesar de que se constata un paulatino incremento del género de los reportajes en los últimos años analizados.

b. Con el paso de los años las piezas informativas publicadas en los anuarios de la agencia SINC son más extensas, tienen más páginas, porque hay más reportajes y no noticias, un género que necesita menos espacio a la hora de su publicación.

c. Los periodistas le dan una gran importancia a los elementos gráficos en la publicación de informaciones sobre ciencia para hacer más atractiva la pieza. Así todas las piezas publicadas contienen algún elemento gráfico, predominando la fotografía frente a otros.

d. Hay una relación directa entre la utilización del género noticia para informaciones cuyo origen es una revista científica, mientras que para los géneros entrevista o reportaje no hay definición tan clara de dónde procede el origen de la información y porqué se publica esa pieza ahora y no antes o después.

e. En cuanto al número de fuentes utilizadas en cada información predomina el uso de una o dos fuentes.

f. Las fuentes personales son las que se utilizan de una forma más numerosa frente a las documentales. Los periodistas elaboran sus informaciones a partir de declaraciones extraídas a los protagonistas de la pieza informativa.

g. Estas fuentes personales son mayoritariamente responsables científicos y del ámbito universitario en los que se está realizando la investigación de la ciencia de la que se habla.

h. Los temas que predominan son las piezas científicas sobre áreas de Física, Química y Matemáticas frente a otras ciencias como las Humanidades y el Arte, que no están tan consideradas dentro del mundo científico.

i. Aunque en el primer anuario se publicó el mismo número de artículos por temas en los siguientes se ha primado los artículos con un claro peso específico en el mundo de las ciencias denominadas como puras.

j. Las piezas informativas sobre ciencia tienen palabras técnicas aunque cada vez su uso va en descenso para una mejor comprensión del texto por parte de los lectores. Predominan los que utilizan 1 o 20 incluso ningún tecnicismo en su texto. 
k. Se explican con definiciones simples los tecnicismos para una mejor comprensión del lenguaje empleado debido a que se trata de un tipo de lenguaje muy específico.

I. El lenguaje utilizado en las piezas informativas es claro porque los tecnicismos se explican con definiciones simples.

\section{Bibliografía}

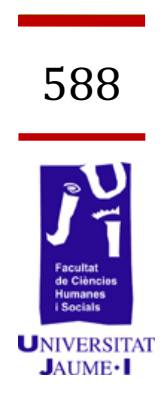

Belenguer Jane, M. (2003). "Información y divulgación científica: dos conceptos paralelos y complementarios en el periodismo científico". En Estudios Sobre el Mensaje Periodístico, no 9, pp. 43-53.

Calvo Hernando, M. (1998). Manual de Periodismo científico. Barcelona. Bosch Casa Editorial

Camiñas Hernández, T. (2002). "La divulgación científica desde las instituciones públicas". En: Comunicar. Revista Científica de Comunicación y Educación, no 19. Grupo Comunicar, 71-76.

CAÑellas, M. J. (2012). “Periodismo científico, el puente entre el laboratorio y la sociedad". En adComunica. Revista Científica de Estrategias, Tendencias e Innovación en Comunicación, $\mathrm{n}$ 4. Castellón: Asociación para el Desarrollo de la Comunicación adComunica, Universidad Complutense de Madrid y Universitat Jaume I, 229-234.

CAsero Ripollés, A. y López Rabadán, P. (2013). La gestión de fuentes informativas como criterio de calidad profesional. En: Gómez Mompart, Josep L; Gutiérrez Lozano, Juan F. y Palau Sampio, Dolors (eds). La calidad periodística. Teorías, investigaciones y sugerencias profesionales. Castelló de la Plana (etc): Publicacions de la Universitat Jaume I (etc).

Cortiñas-Rovira, S. y Ramon-Vegas, X. (2013). "Estrategias de difusión de una revista científica. Un experimento con El Profesional de la Información". En El Profesional de la Información, septiembre-octubre, v. 22, no 5, pp. 405-414.

DE Bustos, R. (2012). "El gabinete de prensa ante el nuevo ecosistema informativo digital". En: Tribuna, 223-227.

De SEMIR, V. (2010). "El mutatis mutandis de la comunicación científica en la era de Internet". En ArtefaCToS, v. 3, no 1, pp. 49-79.

ELíAs, C. (2008). "El periodismo científico como paradigma de la "noticia acatamiento". Una demostración desde las fuentes y una alerta de sus peligros". En: Periodística. № 11, 81-93. 
ELíAS, C. (2009). "La “cultura convergente" y la filosofía Web 2.0 en la reformulación de la comunicación científica en la era del ciberperiodismo". En Arbor Ciencia, Pensamiento y Cultura no 737, pp. 623-634

García Mestres, M. y otros (2012). La percepción social de los principales divulgadores españoles de la ciencia. En: Estudios sobre el mensaje periodístico, Vol 18 n- 2, 757-767

MoReno CASTRO, C. (2010). "La construcción periodística de la ciencia a través de los medios de comunicación social: hacia una taxonomía de la difusión del conocimiento científico". En ArtefaCToS, v. 3, no 1, pp. 109130. 